\title{
Upper extremity peripheral neuropathies: role and impact of MR imaging on patient management
}

\author{
Andreisek, G ; Burg, D ; Studer, A ; Weishaupt, D
}

\begin{abstract}
To investigate the role of MR imaging (MRI) in the evaluation of peripheral nerve lesions of the upper extremities and to assess its impact on the patient management. Fifty-one patients with clinical evidence of radial, median, and/or ulnar nerve lesions and unclear or ambiguous clinical findings had MRI of the upper extremity at $1.5 \mathrm{~T}$. MR images and clinical data were reviewed by two blinded radiologists and a group of three clinical experts, respectively, with regard to radial, median, and/or ulnar nerve, as well as muscle abnormalities. MRI and clinical findings were correlated using Spearman's (p) rank correlation test. The impact of MRI on patient management was assessed by the group of experts and ranked as "major," "moderate," or "no" impact. The correlation of MRI and clinical findings was moderate for the assessment of the median/radial nerve and muscles $(\mathrm{p}=0.51 / 0.51 / 0.63$, respectively) and weak for the ulnar nerve $(\mathrm{p}=0.40)$. The impact of MRI on patient management was assessed as "major" in 24/51 (47\%), "moderate" in 19/51 (37\%), and "no" in 8/51 (16\%) patients. MRI in patients with upper extremity peripheral neuropathies and unclear or ambiguous clinical findings substantially influences the patient management.
\end{abstract}

DOI: https://doi.org/10.1007/s00330-008-0940-y

Posted at the Zurich Open Repository and Archive, University of Zurich ZORA URL: https://doi.org/10.5167/uzh-3610

Journal Article

Published Version

Originally published at:

Andreisek, G; Burg, D; Studer, A; Weishaupt, D (2008). Upper extremity peripheral neuropathies: role and impact of MR imaging on patient management. European Radiology, 18(9):1953-1961.

DOI: https://doi.org/10.1007/s00330-008-0940-y 


\section{Gustav Andreisek \\ Doris Burg \\ Ansgar Studer \\ Dominik Weishaupt}

\section{Upper extremity peripheral neuropathies: role and impact of MR imaging on patient management}

Received: 4 December 2007

Revised: 13 January 2008

Accepted: 3 February 2008

Published online: 28 March 2008

(C) European Society of Radiology 2008
Our study was not supported by industry or any grants.

G. Andreisek · D. Weishaupt

Institute for Diagnostic Radiology,

Department of Medical Radiology,

University Hospital Zurich,

Raemistrasse 100 ,

8091 Zurich, Switzerland

\section{Burg}

Division for Plastic, Hand- and

Reconstructive Surgery, Department of

Surgery, University Hospital Zurich

(Academic Medical Center),

Raemistrasse 100 ,

8091 Zurich, Switzerland

Present address:

D. Burg

Haldenstrasse 14,

8124 Maur, Switzerland

\section{A. Studer}

Department of Neurology,

University Hospital Zurich,

Raemistrasse 100,

8091 Zurich, Switzerland

\section{G. Andreisek $(\bowtie)$}

Department of Medical Imaging,

5th Floor, Mount Sinai Hospital and the

University Health Network,

University of Toronto,

600 University Ave,

Toronto, ON, M5G 1X5, Canada

e-mail: gustav@andreisek.de

Tel.: +1-416-5864800

Fax: +1-416-5868695

\begin{abstract}
To investigate the role of MR imaging (MRI) in the evaluation of peripheral nerve lesions of the upper extremities and to assess its impact on the patient management. Fifty-one patients with clinical evidence of radial, median, and/or ulnar nerve lesions and unclear or ambiguous clinical findings had MRI of the upper extremity at $1.5 \mathrm{~T}$. MR images and clinical data were reviewed by two blinded radiologists and a group
\end{abstract}

of three clinical experts, respectively, with regard to radial, median, and/or ulnar nerve, as well as muscle abnormalities. MRI and clinical findings were correlated using Spearman's ( $p$ ) rank correlation test. The impact of MRI on patient management was assessed by the group of experts and ranked as "major," "moderate," or "no" impact. The correlation of MRI and clinical findings was moderate for the assessment of the median/radial nerve and muscles $(\mathrm{p}=0.51 / 0.51 / 0.63$, respectively) and weak for the ulnar nerve $(p=0.40)$. The impact of MRI on patient management was assessed as "major" in 24/51 (47\%), "moderate" in 19/51 (37\%), and "no" in 8/51 $(16 \%)$ patients. MRI in patients with upper extremity peripheral neuropathies and unclear or ambiguous clinical findings substantially influences the patient management.

Keywords Peripheral nervous system · Peripheral nerves - Magnetic resonance imaging · Upper extremity

\section{Introduction}

The evaluation of peripheral nerve lesions of the upper extremities has traditionally relied on information from an accurate clinical history, a thorough physical examination and electro-diagnostic testing [1]. In most of the patients, these clinical data allow for the determination of location, severity, as well as the etiology of the underlying nerve injury [2].
However, all this clinical information is sometimes insufficient in order to establish the exact diagnosis or with regard to tailoring the treatment for patients with a peripheral nerve lesion. In clinical practice, the localization of a nerve lesion in the absence of abnormal electrodiagnostic findings or diagnosing a focal entrapment neuropathy superimposed on a more general peripheral neuropathy may be challenging [1]. Moreover, clinical information including electro-diagnostic studies may also 
be insufficient for determining resectability of a peripheral nerve mass.

Over the past few years, MR imaging (MRI) has gained increased acceptance as a non-invasive diagnostic adjunct to the clinical evaluation of patients with peripheral nerve lesions [2]. State-of-the-art MRI is not only able to visualize a variety of abnormalities of the nerve itself, but is also useful for the demonstration of denervation changes of the innervated muscles [3, 4]. Recently, it has been shown that MR signal changes in denervated muscles can be used to diagnose peripheral nerve lesions and may even be as accurate as electro-diagnostic examination [5].

Several studies have described typical MRI findings in peripheral nerve lesions of the upper extremities, such as the findings in virgin and recurrent carpal tunnel syndrome $[6,7]$, compressive and entrapment neuropathies of the median, ulnar, and radial nerve at the elbow and at the wrist [8-11], as well in neuropathies of the suprascapular, long thoracic, musculocutaneus, and axillary nerve $[12,13]$. In addition, MRI characteristics of various peripheral nerve tumor masses have been reported [14-16]. Although the MRI findings of these entities are well described, the role of MRI in the evaluation of peripheral nerve lesions remains unclear, especially with regard to the impact of MRI on patient management $[14,17]$.

Hence, the purpose of our paper was to investigate the role of MRI in the evaluation of peripheral nerve lesions of the upper extremities and to assess the impact of MRI on patient management.

\section{Materials and methods}

\section{Subjects}

The local institutional review board approved this study, which was performed in a retrospective manner with written informed patient consent for the retrospective review of clinical and MRI data.

In this study, we included only patients with clinically evident neuropathies of the radial, median, and/or ulnar nerve. Eight hundred and forty-eight cross-reference records of the departments of surgery (division of hand and plastic surgery) and the department of radiology from 2003 to 2006 were reviewed. We identified 51 patients who were referred to our institution for MRI of an upper extremity (MRI of the shoulder/axilla, upper arm, elbow, forearm, wrist, and/or hand) with clinical evidence of a lesion on at least one of the aforementioned nerves and in whom clinical findings were ambiguous or in whom final diagnosis remained unclear. We defined "unclear" or "ambiguous" when after a clinical evaluation, including thorough electro-diagnostic testing, from a clinical point of view, the etiology, the exact anatomic location, the number of the lesions (single vs. multiple lesions causing the nerve injury) was uncertain, or when there was a discrepancy between the symptoms and the clinical findings. Patients were only included when their charts contained complete clinical information including all "pre-MRI" clinical data (clinical history, physical and electrodiagnostic examination), the original MRI report as well as all clinical "postMRI" data (treatment plan, surgical report, follow-up data).

Patients with signs of central nervous system abnormalities, lower cervical radiculopathy, and lower trunk brachial plexopathy were excluded. Patients with abnormalities of the suprascapular, long thoracic, musculocutaneus, or axillary nerve were not included in this study. Patients with symptoms of carpal tunnel syndrome were only included if their symptoms were not sufficiently explained by the clinical findings. Patients with carpal tunnel syndrome usually do not routinely have MRI in our institution unless there is a discrepancy between symptoms and clinical findings. We also did not include patients with acute traumatic peripheral nerve lesions. All patients were referred from a team of two hand surgeons and two specialized neurologists.

The mean age of the 51 patients (female, 29; male, 22) was 42.7 years (age range, 18 to 80 years). In these 51 patients, a total of $51 \mathrm{MR}$ examinations were performed (28 right upper extremities, 23 left upper extremities). In all patients only one anatomical region per extremity was examined.

\section{MRI technique}

All MRI examinations were performed on one of two 1.5-T MR systems [Signa Horizon Signa Excite II Echo Speed Plus or Signa Echospeed HD; General Electrics (GE) Healthcare Systems, Waukesha, WI]. Among the 51 patients with $51 \mathrm{MR}$ examinations, $1 \mathrm{MR}$ examination of the shoulder/axilla (2\%), 2 MR examinations of the upper arm (4\%), 9 MR examinations of the elbow (18\%), 18 MR examinations of the forearm (35\%), and 21 MR examinations of the wrist and hand (41\%) were performed. According to the anatomic region of interest, the upper extremity was positioned in a neutral position in the center of the corresponding custom-designed coil, which was placed in the isocenter of the magnet. A phased array shoulder coil (Medical Advances Inc., Milwaukee, WI) or an eight-channel torso-pelvic coil (GE) was applied for imaging the shoulder/axilla region. A cardiac coil with a four- or eight-channel design (GE) was used for the upper arm and elbow. An extremity array coil (Medrad Inc., Indianola, PA) was applied for the forearm and hand. MRI of the wrist was performed using a high-resolution wrist coil (MRI Devices Corp., Orlando, FL). T1-, T2-, and intermediate-weighted spin echo (SE) or fast spin echo (FSE) as well as T1-weighted short spoiled gradient recalled echo pulse (SSPGR) and short tau inversion recovery (STIR) sequences were acquired in each anatomic region in all three planes. Sequence parameters included 
TR 197-4,840 ms, TE 9-110 ms, TI $150 \mathrm{~ms}$ (for STIR sequences), and an echo train length of 8 (for FSE sequences). In addition, a three-dimensional (3D) gradient echo (GRE) sequence (TR/TE 20/12 ms, flip angle 20) was acquired in the hands and wrists. Other parameters included: section thickness 1.3 to $4.0 \mathrm{~mm}$, intersection gap 0.3 to $1.0 \mathrm{~mm}$, acquisition matrix $256-512 \times 192-256$, field of view $12-30 \times 12-30$, and numbers of excitations 2-4. Contrast enhanced T1-weighted fat saturated SE or SSPGR sequences were acquired after intravenous administration of $0.1 \mathrm{mMol} / \mathrm{kg}$ body weight of Gd-DTPA (Magnevist ${ }^{\mathbb{R}}$; Schering AG, Berlin, Germany) in all patients. All sequences were used for retrospective MRI analysis.

\section{Clinical data}

Before the MRI referral, all 51 patients had a detailed clinical and neurological examination including electrodiagnostic examinations of the radial, median, and ulnar nerve by one of two experienced hand surgeons and by one of two specialized neurologists, respectively. Standard fine-needle electromyography of individual muscles was used in cases with clinical evidence of a muscle abnormality [1]. All electro-diagnostic studies were performed according to the consensus criteria of the American Association of Electro-Diagnostic Medicine (AAEM) [1] and the results were thoroughly recorded in the patient's charts.

\section{MRI analysis}

Two blinded radiologists (G.A.; D.W.) analyzed retrospectively all MR images independently and in a random order. Imaging analysis was performed on a separate workstation (Advantage Windows 4.2; GE).

First, all MR images were evaluated with regard to radial, median, and/or ulnar nerve abnormalities. To facilitate a direct correlation of MRI and clinical findings (see below), we selected a simple binary classification system (adapted from Jarvik et al. [18]): $0=$ normal nerve (normal nerve signal, absence of thinning/thickening, or external nerve compression); 1 =abnormal nerve (abnormal nerve signal, presence of thinning/thickening, external nerve compression, or a mass lesion).

Second, both observers noted all muscle abnormalities, again using a simple binary classification system: $0=$ normal muscle (normal muscle signal, absence of muscle atrophy); 1=abnormal muscle ("muscle edema", presence of muscle atrophy). "Muscle edema" was presumed in the event of high MR signal on T2-weighted or STIR MR images [19]. The MR signal for muscles was considered normal if it was generally much lower than that of fat, slightly higher than that of water on T1-weighted images, and much lower than that of both fat and water on T2weighted images. On STIR MR images, normal muscle signal intensity should be higher than that of fat, but much lower than that of water. Muscle atrophy was defined as followed: moderate muscle atrophy = significant loss of muscle volume $(<50 \%$ of normal muscle volume); severe muscle atrophy $=$ presence of fatty degeneration with $>50 \%$ loss of normal muscle volume $[20,21]$.

Third, both observers had to decide, in cases with the presence of a muscle abnormality, whether it was caused by a myogenic (i.e., the abnormality is caused by a primary affection of the muscle, no specific pattern of involved muscles) or either a neurogenic etiology (i.e., the abnormality is caused by an affection or lesion of the nerve, specific pattern of involved muscles according to their innervations). Fourth, each observer was asked whether the abnormalities could represent either an "acute" ( $<4$ weeks), "subacute" (4 weeks to 1 year), or "chronic" disorder ( $>1$ year). Acute neurogenic muscle disorders may show muscle edema without muscle atrophy, whereas chronic muscle disorders may show muscle atrophy without muscle edema. "Subacute" defines the time range between 4 weeks and 1 year in duration [4, 22] and may be characterized by muscle edema and muscle atrophy at the same time.

Finally, each observer was asked to make a final MR diagnosis based on MRI findings with regard to the location and the underlying etiology of the peripheral nerve lesion.

Clinical correlation and assessment of the impact of MRI on further patient management

Figure 1 illustrates the different steps of the clinical data analysis (Fig. 1), which were organized by the study coordinator (G.A.). Clinical data analysis was performed by a panel of clinical experts (one hand surgeon and two neurologists). In the first step, the study coordinator presented all clinical data from the patient's charts (including clinical history, physical and electro-diagnostic examination) to the clinical experts as far as these examinations were performed prior to MRI ("pre-MRI" clinical data). This means that at step 1 of the review process, the clinical experts were blinded to the original MRI report as well as to all other "post-MRI" clinical data.

During this first step, the clinical experts were asked which of the nerves were affected by the abnormality as well as the presumed location of the abnormality with regard to its anatomical course. For grading nerve and muscle abnormalities, the clinical experts used the same binary classification system that was used by the radiologist to classify nerves and muscle on MR images $(0=$ normal $/ 1=$ abnormal nerve and muscle, respectively). In addition, all clinically present or suspected muscle abnormalities were further classified as "myogenic" or 
"neurogenic." All abnormalities were assigned as an "acute," "subacute," or "chronic" disorder according to the aforementioned definitions of these periods. Finally, based on the "pre-MRI" clinical data, the clinical experts were asked to set a final "clinical diagnosis" for the location and the underlying etiology of the peripheral nerve lesion. The results of this first step of the review process were used for the subsequent correlation of clinical data with the MRI findings (Fig. 1).

The purpose of the second step of the review process was to assess the impact of MRI on the patient management based on all available clinical data. As a difference to step 1 , at this time, the results of the original MRI report and all other "post-MRI" clinical data, including surgical reports and follow-up data, were made available from the patient chart by the study coordinator. The clinical experts noted if the MR examination provided additional information that helped in establishing the final diagnosis (this was called "moderate impact"), or if the MR examination changed the therapeutic considerations, influenced the decision to perform surgery and/or helped in planning the surgery (this was called "major impact").

\section{Statistical analysis}

The interobserver agreement between both radiologists with regard to the MRI analysis was calculated by using the $\kappa$ statistics (Fig. 1). Negative kappa values express discordance of the observations. Kappa values between 0.41 and 0.60 correspond to a moderate agreement, between 0.61 and 0.80 to a substantial agreement, and values higher than 0.80 are considered as excellent interobserver agreement [23].

The correlation of clinical and MRI findings was calculated by using Spearman's (p) rank correlation test. Absolute $p$ values between $0.5<p<0.8$ indicate a moderate strength of correlation, whereas $\mathrm{p}>0.8$ is considered as strong to perfect correlation [24].

Cross table calculations were performed to provide information about the impact of MRI on the patient management as noted with regard to the different causes of the peripheral neuropathies, namely "mass lesions," "entrapment," and "post-trauma/inflammatory conditions." The Kruskal-Wallis test was used to determine if there is a significant difference between these three groups with regard to the impact of MRI on treatment [25].

Statistical analysis and a descriptive analysis were performed by using dedicated software (SPSS, release 11.5, SPSS Inc.; Chicago, IL).

\section{Results}

\section{Clinical findings}

The primary reasons for referring the patients for MRI were an unclear etiology [28 of 51 patients (55\%) (Figs. 2 and 3)], an unclear site in respect to the exact anatomic localization, or number of the lesions (i.e., single vs. multiple lesions causing the nerve injury), 7 of 51 patients (14\%); both unclear, the etiologies and the site/exact anatomic localization/numbers of the lesions, 16 of 51 patients (31\%).

Fig. 1 Diagram illustrates the different steps of data analysis. MR imaging (MRI) data were retrospectively analyzed by two blinded radiologists independently. Interobserver agreement was calculated. Radiological findings were correlated with clinical findings, which were retrospectively analyzed by a panel of experts. The impact of MRI on the patient management was assessed by the same panel experts' based on all clinical findings as documented in the patient charts

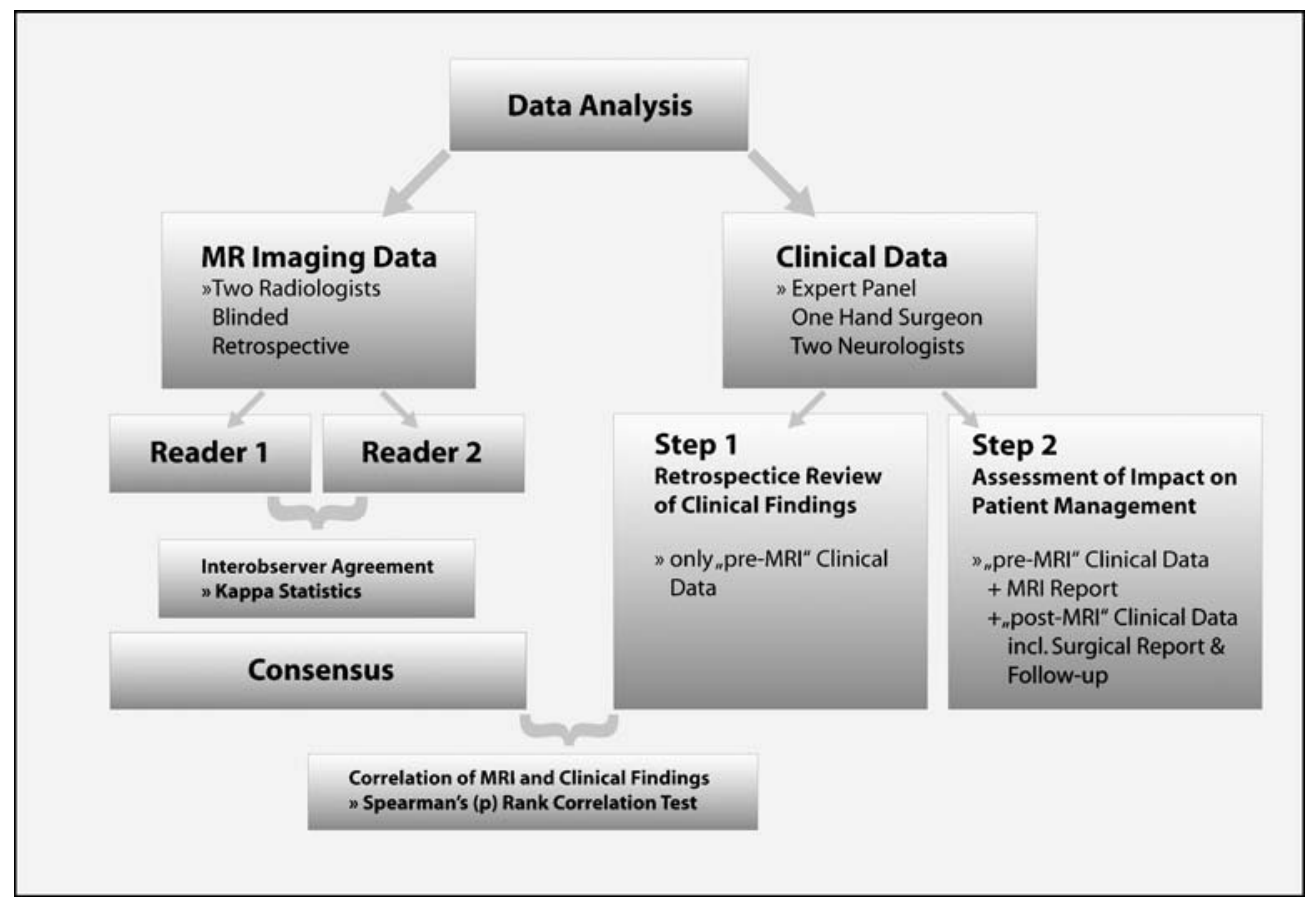




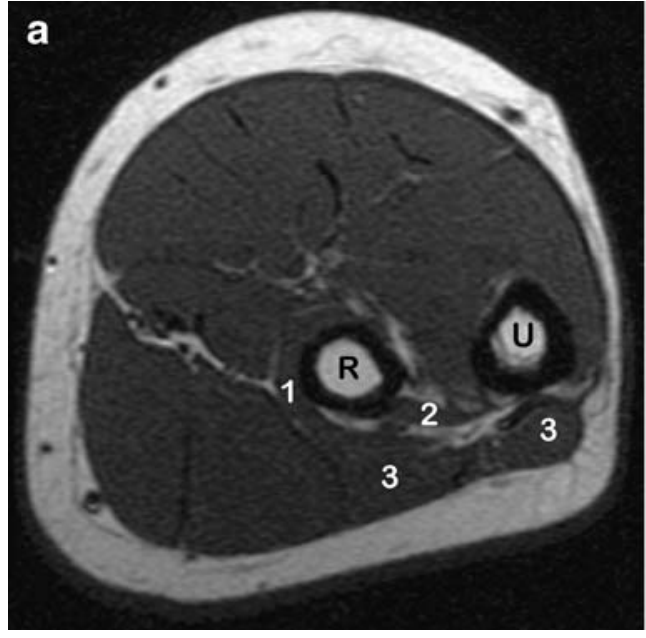

Fig. 2 A 27-year-old female patient with weakness of the extensor muscles of the hand. Axial T1-weighted SE (TR/TE; 400/14 ms) (a) and corresponding short tau inversion recovery (STIR) (TR/TE, 4,840/55 ms; TI, $150 \mathrm{~ms}$ ) (b) MR image at the level of the proximal forearm shows a loss of muscle volume (a) [supinator muscle (m)., 1 ; extensor pollicis brevis and longus m., 2; extensor digitorum, digiti minimi and carpi ulnaris $\mathrm{m} ., 3$ ] and depicts muscle edema (b).

Table 1 shows a summary of the clinical findings in all 51 patients with a clinically evident abnormality of the median, ulnar, and/or radial nerve (step 1 of the clinical data analysis). In all patients, at least one of the aforementioned nerves was affected. Affections of two or all three nerves were seen in 8 of 51 patients $(16 \%)$. Table 2 provides an overview of the final diagnoses as documented in the patient's charts.

\section{MRI findings and clinical correlation}

The MRI findings of 51 patients with a clinically evident abnormality of the median, ulnar, and/or radial nerve are shown in Table 1. Based on MRI findings, nerve abnormalities affecting two or all three aforementioned nerves were present in 6 of 51 patients $(12 \%)$.

Overall, the agreement between both radiologists for MRI analysis was substantial to perfect ( $\kappa=0.74$ to 1.0 ). Detailed kappa values are given as follows: grading of the median nerve, excellent agreement $(\kappa=1.0)$; grading of the ulnar nerve, excellent agreement $(\kappa=0.96)$; grading of the radial nerve, excellent agreement $(\kappa=0.91)$; overall muscle grading, excellent agreement $(\kappa=0.88)$; presence/ absence of muscle atrophy, substantial agreement $(\kappa=$ $0.74)$; presence/absence of muscle edema, excellent agreement $(\kappa=1.0)$; etiology of muscle abnormality, excellent agreement $(\kappa=0.90)$; stage of disorder (acute vs. chronic), excellent agreement $(\kappa=0.94)$.

MRI was able to detect the etiology of nerve disorders in 26 of the 28 patients $(93 \%)$ who were referred for MRI with an unclear etiology. MRI revealed the site, exact anatomic

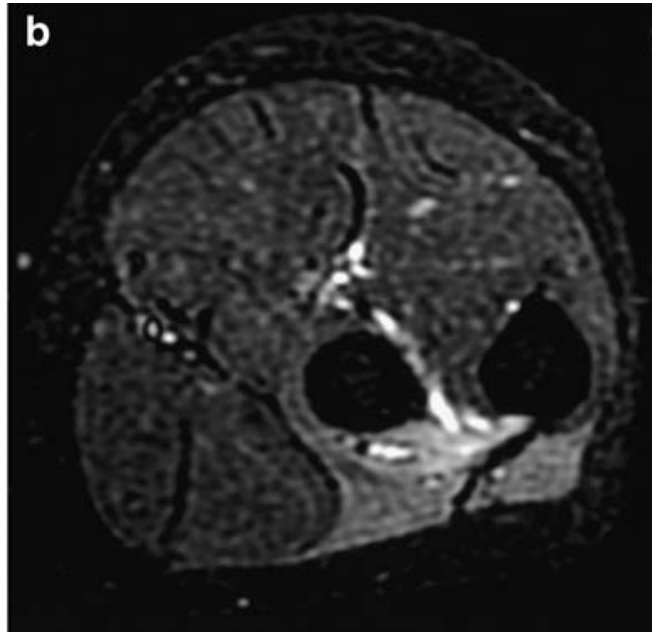

Due to the pattern of muscle involvement (all muscles affected are innervated by the posterior interosseous nerve) with synchronic muscle edema and atrophy, both radiologists diagnosed a subacute posterior interosseous nerve syndrome (PINS) when retrospectively reviewing the MRI data. This radiological diagnosis was identical with the final diagnosis, as documented in the patient chart after surgery
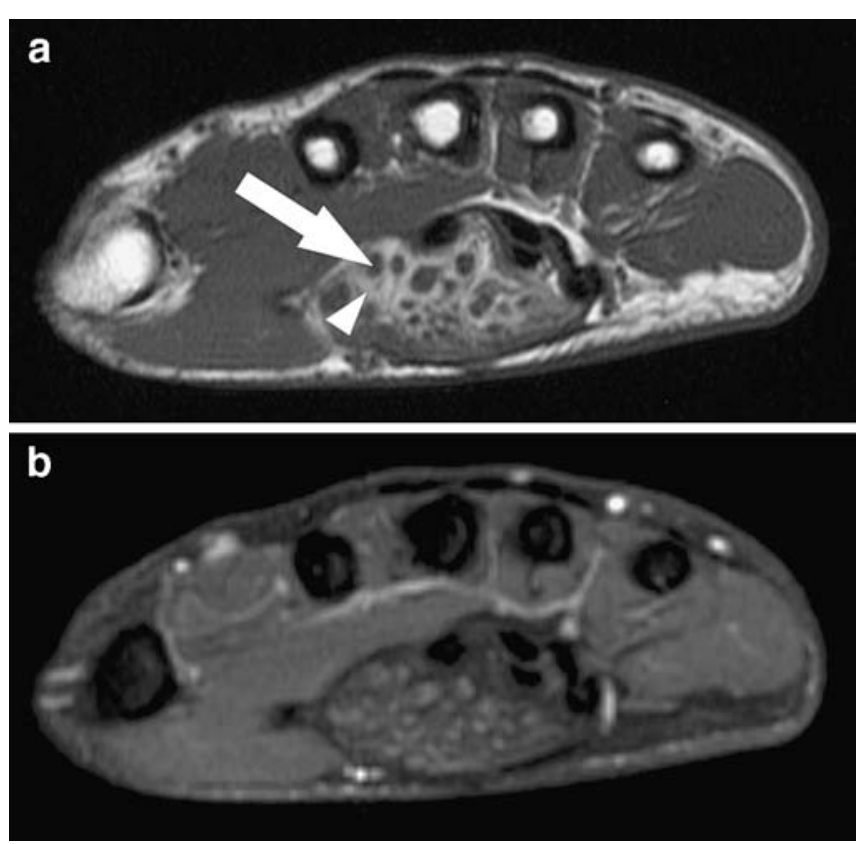

Fig. 3 A 45-year-old male patient with clinical evidence of carpal tunnel syndrome of unknown etiology. Axial T1-weighted SE (TR/ TE 500/9 ms) (a) and corresponding fat-suppressed coronal T2weighted fast spin echo (FSE) (TR/TE, 3,460/110 ms) (b) MR image at the level of the metacarpal bones. Based on the MRI findings of a gross enlargement of the median nerve with a fascicular pattern and fibroadipose tissue (arrowhead) between the individual nerve fascicles (arrow), both radiologists diagnosed a fibrolipomatous hamartoma of the median nerve when retrospectively reviewing the MRI data. This radiological diagnosis was identical with the final diagnosis, as documented in the patient chart after surgery 
Table 1 Clinical findings, MR imaging findings, and correlation of clinical and MR imaging findings in 51 patients with a clinically evident abnormality of the median, ulnar, and/or radial nerve

$\begin{array}{lll}\text { Clinical findings } & \text { MR imaging } & \text { Correlation } \\ \text { no. }(\%) & \text { findings no. }(\%) & (\mathrm{p})\end{array}$

\begin{tabular}{|c|c|c|c|}
\hline Nerves & & & \\
\hline Median nerve & & & \\
\hline Normal & $22(43)$ & 35 (69) & 0.51 \\
\hline Abnormal & $27(57)$ & $16(31)$ & \\
\hline Ulnar nerve & & & \\
\hline Normal & $32(63)$ & $34(67)$ & 0.40 \\
\hline Abnormal & $19(37)$ & 17 (33) & \\
\hline Radial nerve & & & \\
\hline Normal & $42(82)$ & $43(84)$ & 0.51 \\
\hline Abnormal & $9(18)$ & $8(16)$ & \\
\hline Muscles & & & \\
\hline $\begin{array}{l}\text { Normal } \\
\text { muscles }\end{array}$ & $29(57)$ & $28(55)$ & 0.63 \\
\hline $\begin{array}{l}\text { Abnormal } \\
\text { muscles }\end{array}$ & $22(43)$ & $23(45)$ & \\
\hline Atrophy & & & \\
\hline Moderate & $9(17)$ & $5(10)$ & 0.59 \\
\hline Severe & $15(29)$ & 12 (29) & \\
\hline $\begin{array}{l}\text { Muscle } \\
\text { function }\end{array}$ & & & \\
\hline Weakness $^{1}$ & $32(63)$ & n.a. & n.a. \\
\hline $\mathrm{EMG}^{2}$ & $20(39)$ & n.a. & \\
\hline Edema & & & \\
\hline $\mathrm{T} 2 \mathrm{w}$ images & n.a. & 19 (37) & n.a. \\
\hline Etiology & & & \\
\hline Myogenic & $1(2)$ & $3(6)$ & 0.48 \\
\hline Neurogenic & $20(39)$ & $20(39)$ & \\
\hline $\begin{array}{l}\text { Stage of } \\
\text { disorder }\end{array}$ & & & \\
\hline Acute & $9(17)$ & $5(9)$ & 0.48 \\
\hline Subacute & $10(19)$ & $8(16)$ & \\
\hline Chronic & $13(25)$ & $10(20)$ & \\
\hline
\end{tabular}

Data are presented as sum of cases (no.). Numbers of cases are also expressed as percentage of all 51 cases, calculated as follows: percentage $\%=($ no. $/ 51) * 100 .{ }^{1}$ Presence of muscle weakness, graded according to the British Medical Research Council (BMC) grading system $;{ }^{2}$ presence of muscle denervation signs (including fibrillation potentials and positive sharp waves) at fine-needle electromyogram; n.a. = not applicable; $\mathrm{T} 2 \mathrm{w}=\mathrm{T} 2-$ weighted

localization or the number of the lesions (i.e., single vs. multiple lesions causing the nerve injury) in all seven patients $(100 \%)$ in whom the data were unclear before the MR examination. MRI detected multiple lesions in one of these seven patients $(14 \%)$. In those 16 patients in whom both the etiologies as well as the site/exact anatomic localization/number of the lesions were unclear prior to MRI, MRI showed either the etiology or the site/exact anatomic localization/number of lesions in 13 patients $(82 \%)$. In only 3 of 51 patients $(6 \%)$, no additional information was derived from MRI.

Clinical and radiological findings showed a weak to moderate correlation ( $\mathrm{p}=0.48$ to 0.63 ) (Table 1$)$.

\section{Impact on patient management}

The impact of MRI on the patient management was described as a "major impact" in 24 of 51 patients $(47 \%$, Fig. 4$)$ and as "moderate impact" in 19 of 51 patients $(37 \%)$. In 8 of 51 patients $(16 \%)$, there was "no impact" of MRI on patient management. A sub-analysis (Table 3) with respect to the impact of MRI on patient management and different causes of neuropathies ("mass lesions," "entrapment" and "post-trauma/ inflammatory conditions") showed no significant difference (Kruskal-Wallis test, $\mathrm{p}=0.56$ ) among these three groups.

\section{Discussion}

Our study illustrates the role of MRI in the evaluation of upper extremity peripheral nerve lesions, which is not well

Table 2 Final diagnosis (as documented in the patient charts) in 51 patients with a clinically evident abnormality of the median, ulnar and/or radial nerve

\begin{tabular}{ll}
\hline Diagnosis & No. $(\%)$ \\
\hline Mass lesions (n=29) & \\
Neurofibroma & $8(16)$ \\
Fibrolipomatous hamartoma & $6(12)$ \\
Schwannoma & $2(4)$ \\
Nerve sheet ganglia & $2(4)$ \\
Neurosarkoma & $1(2)$ \\
Entrapment (n=25) & \\
Anterior interosseus nerve syndrome & $5(10)$ \\
Posterior interosseus nerve (supinator) syndrome & $5(10)$ \\
Cubital tunnel syndrome & $7(13)$ \\
Carpal tunnel syndrome & $4(8)$ \\
Guyon canal syndrome & $2(4)$ \\
Pronator syndrome & $1(2)$ \\
External compression due to lipoma & $1(2)$ \\
Post-trauma/inflammatory conditions (n=7) & \\
Scarring & $3(6)$ \\
Multifocal motor neuropathy (MMN) & $2(4)$ \\
Polyneuropathies & $2(4)$ \\
\hline
\end{tabular}

Data are presented as sum of cases (no.). Numbers of cases are also expressed as percentage of all 51 cases, calculated as follows: percentage $\%=($ no. $/ 51) * 100$ 

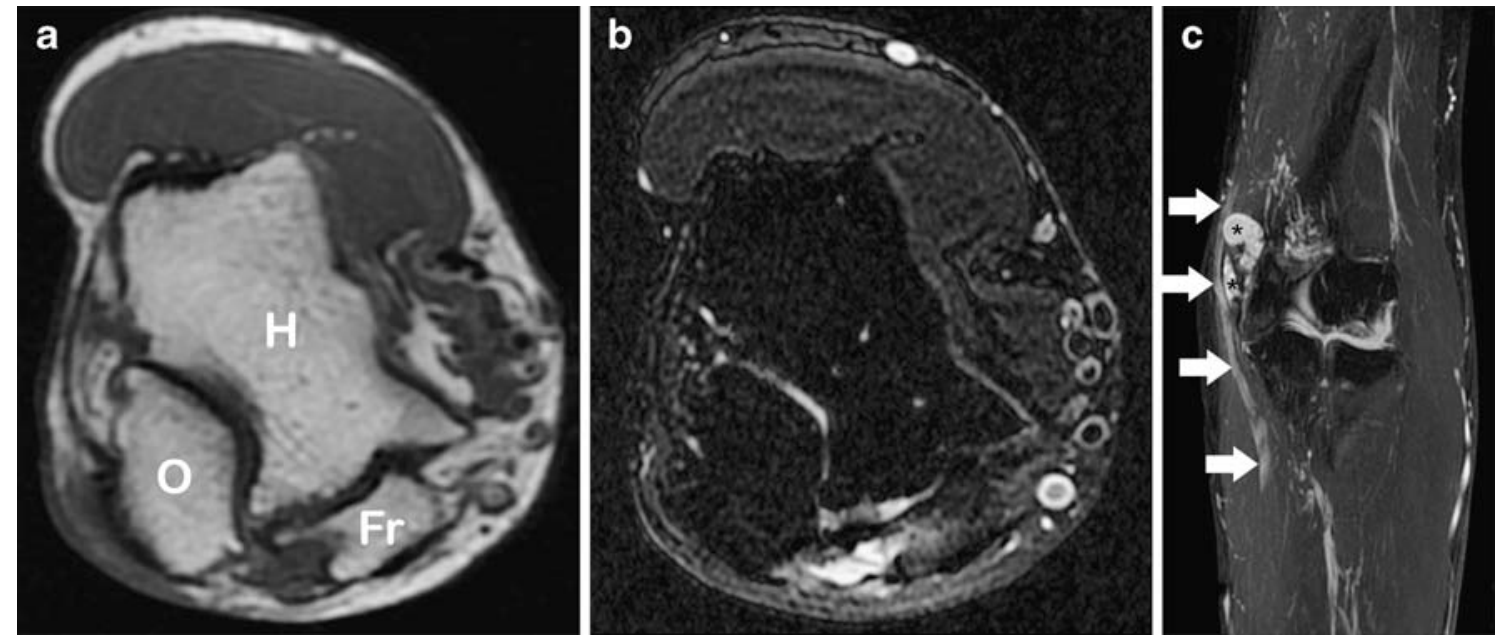

Fig. 4 A 43-year-old male patient who had a motorcycle accident 8 months ago with resulting chronic elbow pain, hand muscle weakness, and a normal electrodiagnostic ulnar nerve test. $(\mathbf{a}, \mathbf{b})$ Axial T1-weighted SE (TR/TE, 560/9 ms) (a) and corresponding STIR (TR/TE, 4,580/35 ms; TI, $150 \mathrm{~ms}$ ) (b) MR images at the level of the elbow reveal a bone fragment (Fr) and increased signal intensity at the medial aspect of the elbow $(\mathrm{H}=$ humerus; $\mathrm{O}=$ olecranon). (c) Thin-slice maximum intensity projection (MIP)

established in literature. Although there is generally consensus that the diagnosis of a peripheral neuropathy is commonly based on clinical and electrodiagnostic studies, some recent articles have shown that MRI may be helpful in establishing the diagnosis $[10,26]$ or may guide further patient management in cases where the clinical findings are unclear or ambiguous $[27,28]$.

Our MRI protocols are mainly focused on the assessment of morphological criteria and do not include functional MRI techniques. When reviewing the literature with regard to MRI of upper extremity peripheral neuropathies, most publications are also focused on the morphological appearance of those neuropathies $[6,9,11,13,17]$. We found only a few recent experimental studies that also addressed the potential of advanced techniques for peripheral nerve

Table 3 Impact of MRI on patient management in 51 patients with a clinically evident abnormality of the median, ulnar, and/or radial nerve-cross table calculation

\begin{tabular}{llll}
\hline$(n=51)$ & $\begin{array}{l}\text { Mass lesions } \\
(n=19)\end{array}$ & $\begin{array}{l}\text { Entrapment } \\
(\mathrm{n}=25)\end{array}$ & $\begin{array}{l}\text { Post-trauma/ } \\
\text { inflammatory } \\
\text { conditions }(\mathrm{n}=7)\end{array}$ \\
\hline
\end{tabular}

\section{Major impact \\ $\mathrm{n}=24 \quad 8$}

Moderate impact

$\mathrm{n}=19 \quad 4$

10

No impact

$\mathrm{n}=7$

4
3

2

2 image created from the STIR MR data set shows a multi-lobulated mass (asterisks) and an increased signal intensity of the ulnar nerve (arrows). Based on the MRI findings, both radiologists diagnosed a focal ulnar neuritis, a ganglion, and a bone fragment when retrospectively reviewing the MRI data. This radiological diagnosis was identical with the final diagnosis, as documented in the patient chart after surgery

imaging including diffusion-direction-dependent (DDI) MRI [29] as well the use of newer MR contrast agents [such as superparamagnetic iron oxide (SPIO) particles or gadofluorine M] [30, 31]. However, regardless the imaging protocols used, to the best of our knowledge, none of these studies systematically evaluated the role of MRI and its impact on patient management in upper extremity peripheral neuropathies.

The correlation between clinical findings and MRI in patients with peripheral neuropathies is difficult. In a prospective study by Jarvik et al. on patients with suspected carpal tunnel syndrome (CTS), MRI revealed only a 39\% specificity when compared to clinical examinations including electrodiagnostic studies [18]. In our study, which not only focused on CTS but included a broad variety of upper extremity peripheral neuropathies, we found a weak or moderate correlation between MRI and the clinical findings. The correlation was particularly weak where only direct signs of nerve abnormalities, such as nerve thickening or high signal intensity, were evaluated with MRI. The correlation improved when secondary signs of peripheral neuropathies, such as neurogenic muscle edema and atrophy, were included in the imaging analysis.

A weak or moderate correlation between clinical and MRI findings may not be surprising since MRI and clinical examinations evaluate different aspects of the nerve disturbance. Clinical evaluations mostly address nerve function, while MRI evaluations primarily rely on morphologic criteria. This may explain why most patients are referred for a MRI only when additional anatomic imaging is needed [27], e.g., to reveal a mass, or when clinical findings are ambiguous or unclear [28]. 
Another reason to use MRI in patients with peripheral neuropathies is the potential to serve as an adjunct in the diagnosis and management of these patients. In a series of 12 patients with ulnar nerve entrapment at the level of the elbow, Britz et al. reported that MRI was able to detect two or more neuropathies in patients who had normal electrodiagnostic examinations [32]. In another study by Grant et al., MRI was found to be more sensitive in identifying pathologies of the ulnar nerve at the elbow when compared to pre- or intraoperative electrodiagnostic studies [33].

In our selected group of patients with peripheral neuropathies of the upper extremity, we found that in $84 \%$ of all patients referred with unclear or ambiguous findings, MRI had a "moderate" or "major" impact on therapy. In almost half of the patients, the therapy plan was changed after the MRI, and in the remaining patients, MRI revealed important information that was used for further treatment, such as the planning the duration of conservative treatment and/or physiotherapy. In only $8 / 51$ patients (16\%), the information derived from MRI was not considered as helpful for further patient management. When grouping the patients according to the different causes of peripheral neuropathies (mass lesions, entrapment, and post-trauma/inflammatory conditions), the impact of MRI on the patient management was the same with no statistically significant differences between these groups.

In the future, it may be hypothesized that MRI may also help to predict which patients respond best to medical or surgical treatment. However, additional work is necessary to prospectively determine the ability of MRI to select patients who will or will not benefit from either treatment.

We acknowledge several limitations of this study. The study was performed in a retrospective manner in a highly selected group of patients referred from very experienced clinicians. Since many of the patients were treated conservatively, there was no definite standard of reference. Another limitation may relate to the fact that the clinicians in the panel who performed the retrospective analysis were also the referring physicians.

In conclusion, this study demonstrates that MRI in patients with upper extremity peripheral neuropathies and unclear or ambiguous findings in clinical examinations influences the patient management in a substantial percentage of cases.

Acknowledgements The authors thank Mrs. Anna Mueller-Gerber for her help in preparing the manuscript as well as Prof. Viktor Meyer for his thoughtful comments.

\section{References}

1. Day JW (2001) Electrodiagnosis in diseases of nerve and muscle: principles and practice. In: Kimura J (ed) Neuromuscul disord. Oxford University Press, Oxford

2. Koltzenburg M, Bendszus M (2004) Imaging of peripheral nerve lesions. Curr Opin Neurol 17:621-626

3. McDonald CM, Carter GT, Fritz RC, Anderson MW, Abresch RT, Kilmer DD (2000) Magnetic resonance imaging of denervated muscle: comparison to electromyography. Muscle Nerve 23:1431-1434

4. West GA, Haynor DR, Goodkin R et al (1994) Magnetic resonance imaging signal changes in denervated muscles after peripheral nerve injury. Neurosurgery 35:1077-1085, discussion 1085-1076

5. Bendszus M, Wessig C, Reiners K, Bartsch AJ, Solymosi L, Koltzenberg M (2003) MR imaging in the differential diagnosis of neurogenic foot drop. AJNR Am J Neuroradiol 24:12831289
6. Jarvik JG, Yuen E, Kliot M (2004) Diagnosis of carpal tunnel syndrome: electrodiagnostic and MR imaging evaluation. Neuroimaging Clin N Am $14: 93-102$, DOI $10.1016 / j$. nic. 2004.02.002

7. Wu HT, Schweitzer ME, Culp RW (2004) Potential MR signs of recurrent carpal tunnel syndrome: initial experience. J Comput Assist Tomogr 28:860864

8. Andreisek G, Crook DW, Burg D, Marincek B, Weishaupt D (2006) Peripheral neuropathies of the median, radial, and ulnar nerves: MR imaging features. Radiographics 26 : 1267-1287, DOI 10.1148/ rg. 265055712

9. Beltran J, Rosenberg ZS (1994) Diagnosis of compressive and entrapment neuropathies of the upper extremity: value of MR imaging. AJR Am J Roentgenol 163:525-531

10. Ferdinand BD, Rosenberg ZS, Schweitzer ME et al (2006) MR imaging features of radial tunnel syndrome: initial experience. Radiology 240:161-168, DOI 10.1148/radiol.2401050028
11. Kim S, Choi JY, Huh YM et al (2007) Role of magnetic resonance imaging in entrapment and compressive neuropathy-what, where, and how to see the peripheral nerves on the musculoskeletal magnetic resonance image: part 2. Upper extremity. Eur Radiol 17:509522, DOI 10.1007/s00330-006-0180-y

12. Demondion X, Bacqueville E, Paul C, Duquesnoy B, Hachulla E, Cotten A (2003) Thoracic outlet: assessment with MR imaging in asymptomatic and symptomatic populations. Radiology 227:461468, DOI 10.1148/radiol.2272012111

13. Spinner RJ, Amadio PC (2003) Compressive neuropathies of the upper extremity. Clin Plast Surg 30:155-173 vi

14. Hems TE, Burge PD, Wilson DJ (1997) The role of magnetic resonance imaging in the management of peripheral nerve tumours. J Hand Surg [Br] 22:57-60

15. Murphey MD, Smith WS, Smith SE Kransdorf MJ, Temple HT (1999) From the archives of the AFIP. Imaging of musculoskeletal neurogenic tumors: radiologic-pathologic correlation. Radiographics 19:1253-1280 
16. Toms AP, Anastakis D, Bleakney RR, Marshall TJ (2006) Lipofibromatous hamartoma of the upper extremity: a review of the radiologic findings for 15 patients. AJR Am J Roentgenol 186:805-811, DOI 10.2214/ AJR.04.1717

17. Spratt JD, Stanley AJ, Grainger AJ, Hide IG, Campbell RS (2002) The role of diagnostic radiology in compressive and entrapment neuropathies. Eur Radiol 12:2352-2364, DOI 10.1007/ s00330-001-1256-3

18. Jarvik JG, Yuen E, Haynor DR et al (2002) MR nerve imaging in a prospective cohort of patients with suspected carpal tunnel syndrome. Neurology 58:1597-1602

19. May DA, Disler DG, Jones EA, Balkissoon AA, Manaster BJ (2000) Abnormal signal intensity in skeletal muscle at MR imaging: patterns, pearls, and pitfalls. Radiographics 20(Spec No):S295-S315

20. Fuchs B, Weishaupt D, Zanetti M, Hodler J, Gerber C (1999) Fatty degeneration of the muscles of the rotator cuff: assessment by computed tomography versus magnetic resonance imaging. J Shoulder Elbow Surg 8:599605
21. Goutallier D, Postel JM, Bernageau J, Lavau L, Voisin MC (1994) Fatty muscle degeneration in cuff ruptures. Pre- and postoperative evaluation by CT scan. Clin Orthop Relat Res:78-83

22. Wessig C, Koltzenburg M, Reiners K, Solymosi L, Bendszus M (2004) Muscle magnetic resonance imaging of denervation and reinnervation: correlation with electrophysiology and histology. Exp Neurol 185:254-261

23. Kundel HL, Polansky M (2003) Measurement of observer agreement. Radiology 228:303-308, DOI 10.1148/ radiol.2282011860

24. Zou KH, Tuncali K, Silverman SG (2003) Correlation and simple linear regression. Radiology 227:617-622, DOI 10.1148/radiol.2273011499

25. Kruskal W (1978) Dickens on statistics. Science 199:1026, DOI 10.1126/ science.199.4333.1026

26. Lacour-Petit MC, Lozeron P, Ducreux D (2003) MRI of peripheral nerve lesions of the lower limbs. Neuroradiology 45:166-170, DOI 10.1007/ s00234-002-0932-6

27. Grant GA, Goodkin R, Maravilla KR, Kliot M (2004) MR neurography: diagnostic utility in the surgical treatment of peripheral nerve disorders. Neuroimaging Clin N Am 14:115-133, DOI 10.1016/j.nic.2004.02.003
28. Weig SG, Waite RJ, McAvoy K (2000) MRI in unexplained mononeuropathy. Pediatr Neurol 22:314-317

29. Skorpil M, Engstrom M, Nordell A (2007) Diffusion-direction-dependent imaging: a novel MRI approach for peripheral nerve imaging. Magn Reson Imaging 25:406-411, DOI 10.1016/j. mri.2006.09.017

30. Bendszus M, Stoll G (2003) Caught in the act: in vivo mapping of macrophage infiltration in nerve injury by magnetic resonance imaging. J Neurosci 23:10892-10896

31. Wessig C, Bendszus M, Stoll G (2007) In vivo visualization of focal demyelination in peripheral nerves by gadofluorine M-enhanced magnetic resonance imaging. Exp Neurol 204:14-19, DOI 10.1016/j. expneurol.2006.09.022

32. Britz GW, Haynor DR, Kuntz C et al (1996) Ulnar nerve entrapment at the elbow: correlation of magnetic resonance imaging, clinical, electrodiagnostic, and intraoperative findings. Neurosurgery 38:458-465, discussion 465

33. Grant GA, Britz GW, Goodkin R, Jarvik JG, Maravilla K, Kliot M (2002) The utility of magnetic resonance imaging in evaluating peripheral nerve disorders. Muscle Nerve 25:314-331 